\title{
Native point defects and their implications for the Dirac point gap at $\mathrm{MnBi}_{2} \mathrm{Te}_{4}(0001)$
}

\author{
M. Garnica $\left(1 D^{1 凶}\right.$, M. M. Otrokov (iD ${ }^{2,3 凶}$, P. Casado Aguilar ${ }^{1,4}$, I. I. Klimovskikh (iD ${ }^{5}$, D. Estyunin (iD ${ }^{5}$, Z. S. Aliev ${ }^{6,7}$, I. R. Amiraslanov ${ }^{7,8}$, \\ N. A. Abdullayev 7,8 , V. N. Zverev ${ }^{9}$, M. B. Babanly 8,10 , N. T. Mamedov ${ }^{7}$, A. M. Shikin ${ }^{5}$, A. Arnau ${ }^{2,11,12}$, A. L. Vázquez de Parga (iD ${ }^{1,4,13,14}$, \\ E. V. Chulkov $\mathbb{D}^{5,11,12,15 凶}$ and R. Miranda $\mathbb{1}^{1,4,13,14 凶}$
}

We study the surface crystalline and electronic structures of the antiferromagnetic topological insulator $\mathrm{MnBi}_{2} \mathrm{Te}_{4}$ using $s c a n n i n g$ tunneling microscopy/spectroscopy (STM/S), micro( $\mu$ )-laser angle-resolved photoemission spectroscopy (ARPES), and density functional theory calculations. Our STM images reveal native point defects at the surface that we identify as $\mathrm{Bi}_{\mathrm{Te}}$ antisites and $\mathrm{Mn}_{\mathrm{Bi}}$ substitutions. Bulk X-ray diffraction further evidences the presence of the $\mathrm{Mn}$-Bi intermixing. Overall, our characterizations suggest that the defects concentration is nonuniform within crystals and differs from sample to sample. Consistently, the ARPES and STS experiments reveal that the Dirac point gap of the topological surface state is different for different samples and sample cleavages, respectively. Our calculations show that the antiparallel alignment of the $\mathrm{Mn}_{\mathrm{Bi}}$ moments with respect to those of the Mn layer can indeed cause a strong reduction of the Dirac point gap size. The present study provides important insights into a highly debated issue of the $\mathrm{MnBi}_{2} \mathrm{Te}_{4}$ Dirac point gap.

npj Quantum Materials (2022)7:7 ; https://doi.org/10.1038/s41535-021-00414-6

\section{INTRODUCTION}

The interplay between magnetism and topology is a fertile ground for exotic ground states in condensed matter ${ }^{1}$. In this context, intrinsic magnetic topological insulators (TIs) have attracted a great deal of attention ${ }^{2-26}$ due to the recent discovery of the first representative of this class, i.e., the van der Waals antiferromagnetic (AFM) compound $\mathrm{MnBi}_{2} \mathrm{Te}_{4}{ }^{7-11}$. This material crystallizes in the trigonal $R \overline{3} m$-group structure ${ }^{27-29}$, made of septuple layer (SL) blocks, in which atomic layers are stacked in the Te-Bi-Te-Mn-Te$\mathrm{Bi}$-Te sequence, as shown in Fig. 1a. Neighboring SLs are bound by van der Waals forces. Below $25 \mathrm{~K}, \mathrm{MnBi}_{2} \mathrm{Te}_{4}$ orders antiferromagnetically due to the antiparallel alignment between alternate, ferromagnetically-ordered Mn layers ${ }^{7,13,30}$, with the local moments pointing out-of-plane (Fig. 1a). The combination of these crystalline and magnetic structures makes $\mathrm{MnBi}_{2} \mathrm{Te}_{4}$ invariant with respect to the $S=\Theta T_{1 / 2}$-symmetry (where $\Theta$ is time-reversal and $T_{1 / 2}$ is a primitive-lattice translation), which gives rise to the $Z_{2}$ topological classification of AFM insulators ${ }^{31,32}\left(Z_{2}=1\right.$ for this material $\left.{ }^{7,9,10}\right)$. According to the bulk-boundary correspondence principle, the topological surface state appears in the bulk bandgap of a Tl, which in the case of the AFM TI might be gapped at the $S$-breaking crystal termination ${ }^{31,32}$. For $\mathrm{MnBi}_{2} \mathrm{Te}_{4}$, the S-breaking surface is (0001), which, according to ab initio calculations, is indeed gapped due to the uncompensated out-ofplane FM Mn layer ${ }^{7,9,10}$. A plethora of exotic phenomena can be hosted if the Fermi level of the experimentally synthesized samples lies inside the Dirac point (DP) gap, such as various kinds of the quantized Hall effect ${ }^{31,33-36}$, axion insulator states $25,37,38$, Majorana fermions ${ }^{39}$, chiral hinge modes ${ }^{40}$, etc.

The experimental studies of the $\mathrm{MnBi}_{2} \mathrm{Te}_{4}$ surface electronic structure have reported contradictory results, with some groups finding a gapped Dirac cone (gap of at least $60 \mathrm{meV}$ and larger) $)^{7,12,14,29,41}$, in agreement with theoretical predictions ${ }^{7,9,10}$, while others revealing a gapless topological surface state ${ }^{42-47}$. A recent photoemission study reports a reduced DP gap of about $20 \mathrm{meV}$ in some $\mathrm{MnBi}_{2} \mathrm{Te}_{4}$ samples ${ }^{48}$. Given the complex crystal structure of $\mathrm{MnBi}_{2} \mathrm{Te}_{4}$, the problem may lie in whether the bulk and/or surface of real samples faithfully reproduce the predicted properties of the ideal crystal structure (shown in Fig. 1a-c), in particular, the magnetic ones. This is especially important in view of the possible applications since the envisaged quantum devices $^{49,50}$ would often employ few SL-layer thick films.

In this paper, we report on a combined study of the AFM TI $\mathrm{MnBi}_{2} \mathrm{Te}_{4}(0001)$ surface using low-temperature scanning tunneling microscopy/spectroscopy (STM/S), high-resolution micro( $\mu$ )-laser angle-resolved photoemission spectroscopy (ARPES), and density functional theory (DFT) calculations. High-resolution STM images complemented by the STM simulations allow us to observe, identify and provide a detailed characterization of two types of point defects: the $\mathrm{Bi}_{\mathrm{Te}}$ antisites (i.e., $\mathrm{Bi}$ atoms at the Te sites) located in the surface layer and $\mathrm{Mn}_{\mathrm{Bi}}$ substitutions ( $\mathrm{Mn}$ atoms at the $\mathrm{Bi}$ sites) in the second atomic layer. The fingerprints of these defects appear as circular protrusions and triangular depressions, respectively, and are readily seen in the topographic images at

\footnotetext{
'Instituto Madrileño de Estudios Avanzados en Nanociencia (IMDEA-Nanociencia), 28049 Madrid, Spain. ${ }^{2}$ Centro de Física de Materiales (CFM-MPC), Centro Mixto CSIC-UPV/ EHU, 20018 Donostia-San Sebastián, Basque Country, Spain. ${ }^{3}$ IKERBASQUE, Basque Foundation for Science, 48011 Bilbao, Spain. ${ }^{4}$ Departamento de Física de la Materia Condensada, Universidad Autónoma de Madrid, 28049 Madrid, Spain. ${ }^{5}$ Saint Petersburg State University, 198504 Saint Petersburg, Russia. ${ }^{6}$ Azerbaijan State Oil and Industry University, AZ1010 Baku, Azerbaijan. ${ }^{7}$ Institute of Physics, National Academy of Sciences of Azerbaijan, AZ1143 Baku, Azerbaijan. ${ }^{8}$ Baku State University, AZ1148 Baku, Azerbaijan. ${ }^{9}$ Institute of Solid State Physics, Russian Academy of Sciences, Chernogolovka, Russia. ${ }^{10}$ Institute of Catalysis and Inorganic Chemistry, Azerbaijan National Academy of Science, AZ1143 Baku, Azerbaijan. " ${ }^{11}$ Departamento de Polímeros y Materiales Avanzados: Física, Química y Tecnología, Facultad de Ciencias Químicas, Universidad del País Vasco UPV/EHU, 20080 Donostia-San Sebastián, Basque Country, Spain. ${ }^{12}$ Donostia International Physics Center (DIPC), 20018 Donostia-San Sebastián, Basque Country, Spain. ${ }^{13}$ Instituto Nicolás Cabrera, Universidad Autónoma de Madrid, 28049 Madrid, Spain. ${ }^{14}$ Condensed Matter Physics Center (IFIMAC), Universidad Autónoma de Madrid, 28049 Madrid, Spain. ${ }^{15}$ Tomsk State University, 634050 Tomsk, Russia. ${ }^{凶}$ email: manuela.garnica@imdea.org; mikhail.otrokov@gmail.com; evguenivladimirovich.tchoulkov@ehu.eus; rodolfo.miranda@imdea.org
} 


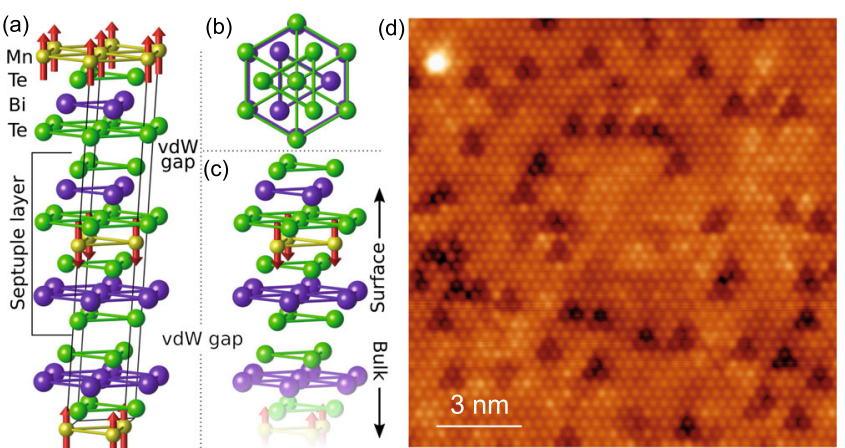

Fig. $1 \mathrm{MnBi}_{2} \mathrm{Te}_{\mathbf{4}}$ bulk and surface structure. a Side view of the bulk crystal structure of $\mathrm{MnBi}_{2} \mathrm{Te}_{4}$ with red arrows showing the interlayer AFM order. The crystal cleavage in this block-layered compound takes place at the van der Waals gap, thus exposing a Te layer of an SL at the surface. b Top and $\mathbf{c}$ side views of the surface crystal structure. d Atomically-resolved STM image of the surface of the cleaved sample ( $1 \mathrm{~V}$ and $0.3 \mathrm{nA}$ ) showing dark triangular depressions and a bright circular protrusion.

relatively large bias voltages (Fig. $1 \mathrm{~d}$ ). The presence of the $\mathrm{Mn}_{\mathrm{Bi}}$ substitutions in the second layer strongly suggests that they should also occur in the sixth (Bi) layer as well, while $\mathrm{Bi}$ atoms, in turn, should occupy $M n$ positions in the fourth layer $\left(\mathrm{Bi}_{\mathrm{Mn}}\right)$. This is indeed confirmed by our structure refinement of the X-ray diffraction data.

Importantly, since the defects in the second, fourth, and sixth layers essentially involve $\mathrm{Mn}$, they cause deviations of the magnetic structure from the ideal one (shown in Fig. $1 \mathrm{a}-\mathrm{c}$ ) to a ferrimagnetic ${ }^{51-53}$, which might influence the DP gap size. In line with this, our STS measurements reveal that, depending on the sample cleavage, the local density of states is compatible with both large $(\sim 50 \mathrm{meV})$ and small $(<20 \mathrm{meV})$ DP gaps, in agreement with the laser-ARPES experiments, detecting that the DP gap changes from sample to sample. Our DFT surface electronic structure calculations show that the $\mathrm{Mn}_{\mathrm{Bi}}$ defects cause a strong reduction of the $\mathrm{MnBi}_{2} \mathrm{Te}_{4}$ DP gap due to the antiparallel alignment of the $\mathrm{Mn}_{\mathrm{Bi}}$ moments with respect to those of the $\mathrm{Mn}$ layer ${ }^{51-53}$ and predominant localization of the topological surface state near the Bi layers. We thus attribute the variation of the DP gap in the same sample (as observed by STS) or different samples (ARPES) to a different degree of the defectness of the $\mathrm{MnBi}_{2} \mathrm{Te}_{4}$ crystals at local or global structure level, respectively. This is also supported by the results of our transport measurements that reveal a variation of the Néel temperature in a series of the $\mathrm{MnBi}_{2} \mathrm{Te}_{4}$ single crystal samples. Our results are instrumental in unifying seemingly contradictory reports concerning the $\mathrm{MnBi}_{2} \mathrm{Te}_{4}$ DP gap and stress a necessity of suppressing the cation (Mn-Bi) intermixing thus reducing the number of the $\mathrm{Mn}_{\mathrm{Bi}}$ defects in this AFM TI.

\section{RESULTS}

\section{STM measurements}

Figure $1 d$ shows an atomically-resolved STM image of the $\mathrm{MnBi}_{2} \mathrm{Te}_{4}$ crystal (0001) surface after cleavage in an ultra-high vacuum. A hexagonal lattice with a lateral periodicity of $4.28 \pm$ $0.05 \AA$ is resolved in agreement with the bulk $a$ lattice constant measured by X-ray diffraction ( $a=4.33 \AA$, see Supplementary Information as well as Ref. ${ }^{28}$ ). Since the (0001) plane is the natural cleavage plane containing van der Waals bonded Te layers, the surface is terminated by the outmost Te layer of an SL. On a large scale, see Fig. 2, the surface shows atomically flat terraces several hundreds of $\mathrm{nm}$ in size. They are separated by steps with a height of about $13.7 \pm 0.5 \AA$ (Fig. 2a, b), in good agreement with the expected value of the thickness of a single $\mathrm{MnBi}_{2} \mathrm{Te}_{4} \mathrm{SL}$, which is roughly equal to one-third of the hexagonal $c$ parameter, i.e., $40.93 \AA$ (see Supplementary Information). The topographic STM image of the $\mathrm{MnBi}_{2} \mathrm{Te}_{4}$ flat terrace reveals randomly distributed triangular defects with an average density of the order of $3.4-5.2 \%$ (Fig. 2c and its inset), similar to those at the Te-terminated transition metal dichalcogenides surfaces ${ }^{54}$, as well as bright atomic-size protrusions, although much less abundant.

Atomically-resolved images and their corresponding $\mathrm{d} / \mathrm{d} V$ maps taken at negative (occupied states) and positive (unoccupied states) sample bias voltages $V$ confirm the presence of two types of point defects (labeled as A and B, Fig. 3), randomly distributed over the $\mathrm{MnBi}_{2} \mathrm{Te}_{4}(0001)$ surface. The type $B$ defect, marked with a small dashed triangle, is the most abundant and shows a bias-dependent appearance ${ }^{55}$. At large biases, both negative (Fig. 3a) and positive (Fig. 3b), these defects appear dark and are especially pronounced. However, towards the low bias voltages, e.g. near the Fermi level or in the energy region within the bulk bandgap where the topological surface state is located, these features lose their well-defined triangular shape (Supplementary Figs. 2, 3, and 4). Most clearly, their three-fold symmetry is resolved for $V>0$, as shown in Fig. $3 b$ and inset of Fig. 2c, with the three dark spots located at the positions of surface Te atoms (see also Fig. 1d and Supplementary Figs. 2, 5). Furthermore, the fact that the separation between the three dark spots in a single triangular-shaped depression corresponds to the $\mathrm{MnBi}_{2} \mathrm{Te}_{4}(0001)$ lateral lattice constant $a$ indicates that the defects causing these features are located in the subsurface $(\mathrm{Bi})$ layer. A variation of a bias voltage between -1.7 and $+2.4 \mathrm{~V}$ reveals that the appearance of all dark triangular defects evolves in the same fashion (see Supplementary Fig. 2 for the part of these data in the $[-1.5:+1.5 \mathrm{~V}]$ range). Careful counting allows estimating their concentration in the range $3.4-5.2 \%$ of the Bi sites, depending on the cleavage or surface area (see Supplementary Fig. 6). To get a deeper insight into the nature of the type B defects, we have performed STM simulations using DFT (see Supplementary Information). As seen in Supplementary Fig. 7, the simulated topographic images are consistent with type $\mathrm{B}$ defects being $\mathrm{Mn}_{\mathrm{Bi}}$. Moreover, among all hypothetically possible defects in the $\mathrm{Bi}$ layer, i.e., $\mathrm{Bi}$ vacancy, $\mathrm{Te}_{\mathrm{Bi}}$ antisite or $\mathrm{Mn}_{\mathrm{Bi}}$ substitution, the latter has the lowest formation energy ${ }^{56,57}$. To further verify the point defect behind the triangular-shaped feature, we resort to X-ray diffraction measurements (XRD). They permit to identify a cation disorder in the Mn and Bi positions (Supplementary Fig. 1 and the corresponding note). The structure refinement performed yields the amount of $\mathrm{Mn}$ atoms at $\mathrm{Bi}$ sites of about $4.6 \%$, which is in reasonable agreement with the concentration of second-layer defects seen in our STM measurements as well as results of other $X$-ray diffraction experiments ${ }^{29}$. Moreover, recent neutron diffraction measurements, reported in refs. ${ }^{51,58}$, detect $M_{\mathrm{Bi}}$ atoms, too. Thus, we attribute the triangular-shaped depressions to the $\mathrm{Mn}_{\mathrm{Bi}}$ substitutions in the subsurface layer. Similar features have been previously observed in STM for magnetically doped $\mathrm{Bi}_{2} \mathrm{Se}_{3}$-family $\mathrm{TIs}^{59-61}$ and, recently, for $\mathrm{MnBi}_{2} \mathrm{Te}_{4}(0001)^{13,62,63}$. Besides, the existence of $\mathrm{Mn}_{\mathrm{Bi}}$ defects has also been claimed recently based on the electron energy loss spectroscopy and transmission electron microscopy analysis ${ }^{56}$.

Another atomic-scale defect clearly observed in the STM topographs is a bright circular protrusion, referred to as type A. They are well seen only at relatively large bias voltages, i.e., $V \gtrsim|0.9| \mathrm{V}$ (see Fig. 3a, b and Supplementary Figs. 2, 5). Superimposing a 2D hexagonal lattice on the topograph with atomic resolution yields the lateral location of the defect coinciding with that of the surface Te atoms sites (Fig. 1d). This is consistent with the circular shape of the features, suggesting that they are either incorporated in the surface Te layer or adsorbed on top of it. The bright appearance excludes the possibility of them being Te vacancies, which are usually resolved as depressions at Te-terminated surfaces ${ }^{54,64}$. The small measured 
(a)

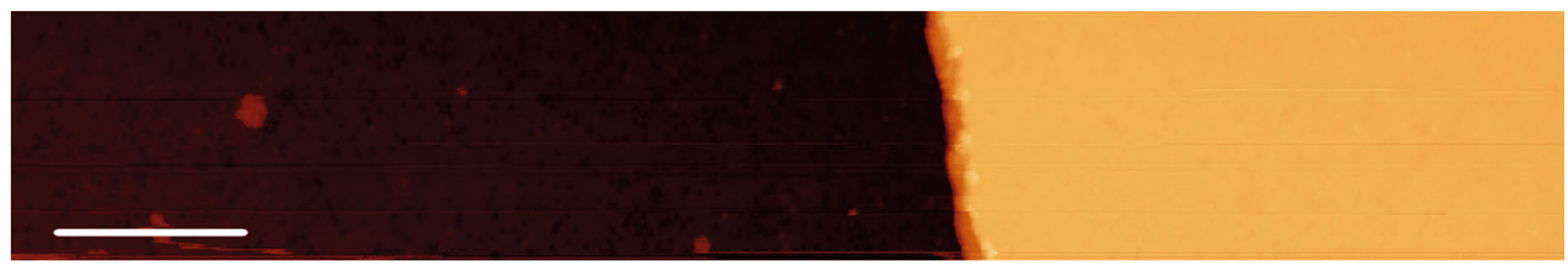

(b)

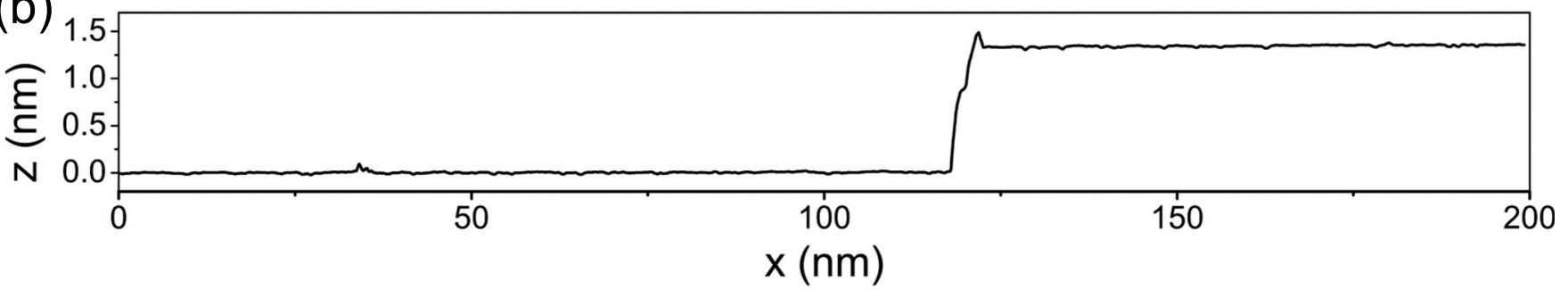

(c)

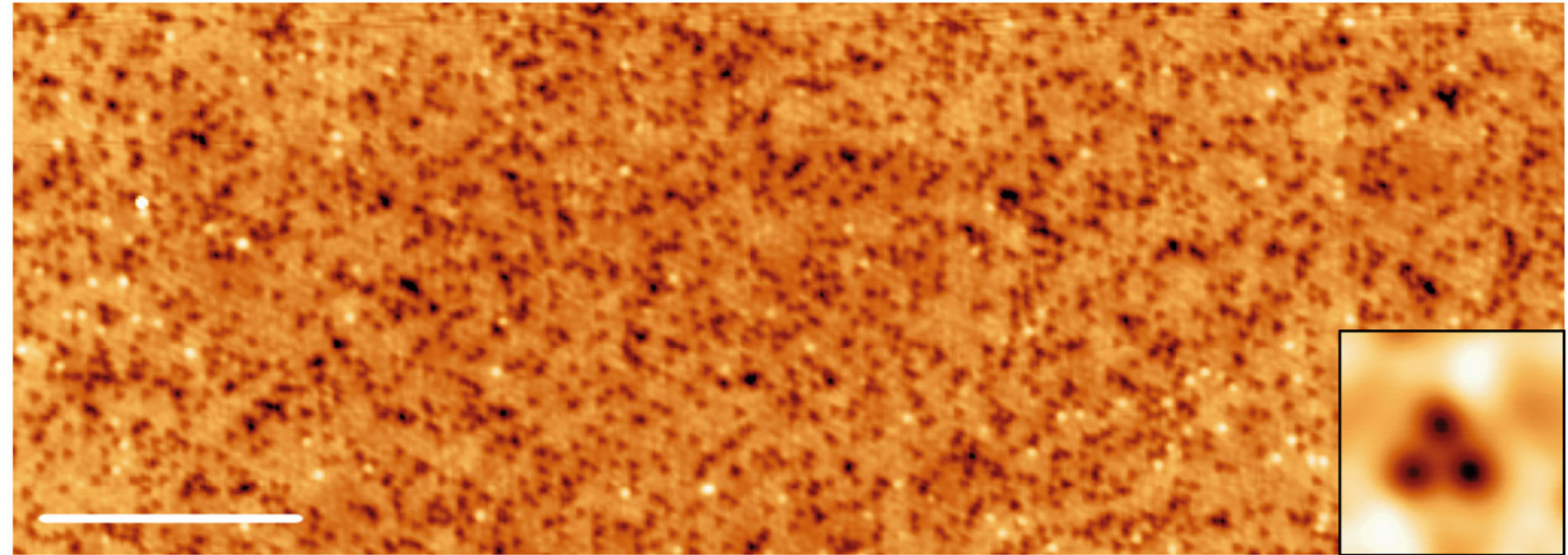

Fig. 2 STM images of the $\mathbf{M n B i}_{2} \mathbf{T e}_{\mathbf{4}}$ surface. Large scale STM topographic images of the $\mathrm{MnBi}_{2} \mathrm{Te}_{4}$ Te-terminated (0001) surface after in situ cleavage showing a flat terraces separated by an SL thick step ( $2 \mathrm{~V}$ and $0.5 \mathrm{nA}$, scale bar $25 \mathrm{~nm})$ and $\mathbf{b}$ its corresponding line profile. $\mathbf{c}$ STM image of a representative area far from the step where the characteristic density of surface defects can be observed ( $1 \mathrm{~V}$ and $0.1 \mathrm{nA}$, scale bar $25 \mathrm{~nm}$ ). Inset: Zoom of $2.5 \mathrm{~nm}^{2}$ of one of the triangular-shaped defects taken at $1 \mathrm{~V}$ and $0.1 \mathrm{nA}$.

apparent height of $0.5 \AA$ at $1 \mathrm{~V}$ as well as the difficulty to manipulate it with the STM tip points towards the substitutional character of this defect. According to recent calculations ${ }^{56,57}$, the lowest formation energy for atomic defects in the van der Waals $\mathrm{Te}$ layers in bulk corresponds to $\mathrm{Bi}_{\mathrm{Te}}$ antisites (Bi atoms substituting the Te atoms), while the formation energies of the $\mathrm{Mn}_{\mathrm{Te}}$ antisite or Te vacancy are much larger. Our STM simulations support the hypothesis of the type $A$ defect being $\mathrm{Bi}_{T e}$, since the feature's appearance as a bright protrusion is reproduced for both occupied and unoccupied states (Supplementary Fig. 7 and the corresponding note). Therefore the type A defects can be identified with the $\mathrm{Bi}_{\mathrm{Te}}$ antisites, similarly to what happens in $\mathrm{Bi}_{2} \mathrm{Te}_{3} \mathrm{Tl}^{64}$, where they also appear as bright protrusions for both bias polarities. This conclusion is in line with previous STM studies of $\mathrm{MnBi}_{2} \mathrm{Te}_{4}(0001)^{13,62,63,65}$. On the basis of our measurements, $\mathrm{Bi}_{\mathrm{Te}}$ appears less frequently than $\mathrm{Mn}_{\mathrm{Bi}}$ (triangular depressions), with an estimated concentration ranging between 0.02 and $0.35 \%$ of the Te layer (depending on the surface location). Such a small concentration does not allow their reliable detection using XRD.

Thus, based on the acquired STM topographic images, we can solidly claim the presence of point defects in the two topmost atomic layers of the $\mathrm{MnBi}_{2} \mathrm{Te}_{4}(0001)$ surface. For the first-layer defect $\mathrm{Bi}_{\mathrm{Te}}$ (type $\left.\mathrm{A}\right), \mathrm{d} / \mathrm{d} \mathrm{d}$ maps show a clear change of contrast when going from $-1.1 \mathrm{~V}$ (occupied states, Fig. $3 \mathrm{c}$ ), where it appears dark, to $1.4 \mathrm{~V}$ (unoccupied states, Fig. 3d), where it looks bright. The same behavior, but inverted, is observed for the $\mathrm{Mn}_{\mathrm{Bi}}$ (type B) defects. Our LDOS simulations reveal the change of contrast for both $\mathrm{Mn}_{\mathrm{Bi}}$ and $\mathrm{Bi}_{\mathrm{Te}}$ (see Supplementary Fig. 8 and the corresponding note), which further supports the defects assignment.

In an attempt to find signatures of the defects lying below the second layer, we focus on the areas where neither first- nor second-layer defects are observed, at least in abundance. Interestingly, even though topographic images do not show any special feature as can be seen in Supplementary Fig. 3, we have observed the appearance of extended bright features in the $\mathrm{d} l / \mathrm{d} V$ maps at -0.4 and $-0.15 \mathrm{~V}$. Their size appears to be approximately equal to nine or six in-plane lattice parameters $a$, respectively. Although based on the STM data it is hardly possible to deduce to which layer the corresponding defects belong, the extension of these features points towards a relatively deep location of the defects.

As it has been mentioned above, our structure refinement based on the XRD measurements indicates the existence of the cation $(\mathrm{Mn}-\mathrm{Bi})$ intermixing, whose signatures are clearly seen in STM as well. The latter means that, apart from the second (subsurface) layer, $\mathrm{Mn}_{\mathrm{Bi}}$ atoms should also occur in the sixth layer counting from the surface, while Bi atoms, in turn, should occupy $\mathrm{Mn}$ positions $\left(\mathrm{Bi}_{\mathrm{Mn}}\right)$ in the fourth layer. However, no clear signatures of the defects lying below the second atomic layer have been observed on the STM topographic images so $\operatorname{far}^{13,62,63,65}$. As far as the spectroscopic $d / / d V$ imaging is concerned, apart from the above discussed deep-lying defects (Supplementary Fig. 3), a feature with a lateral size of $3 a$ has been 
4
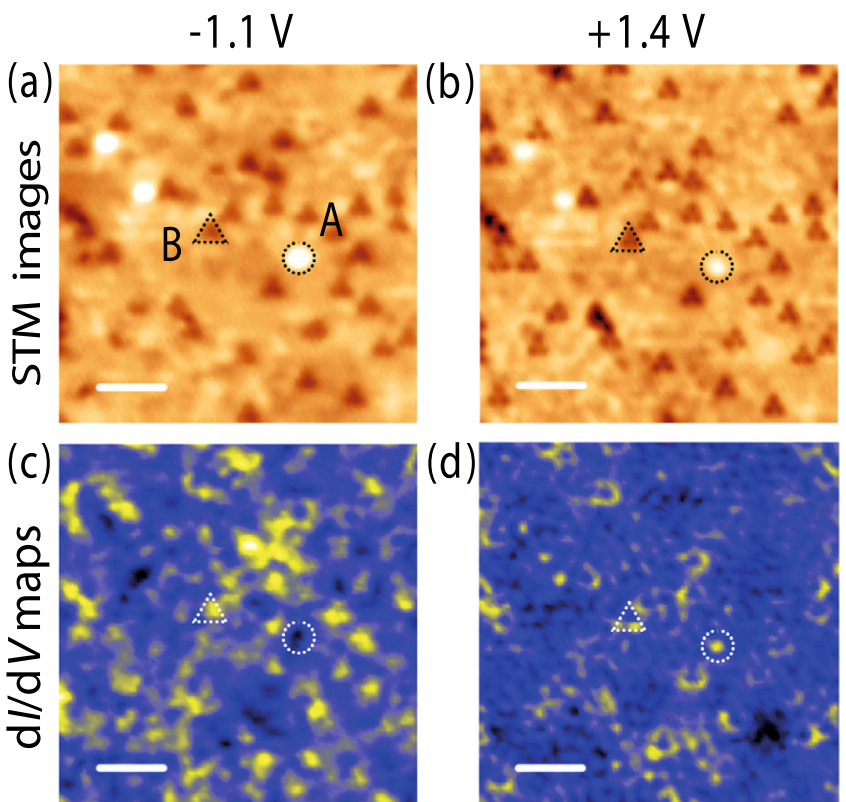

Fig. 3 Atomic resolution STM/S images of native point defects at the $\mathrm{MnBi}_{2} \mathbf{T e}_{\mathbf{4}}$ surface. Topographic STM images were taken at $1.2 \mathrm{~K}$ on the same area showing $\mathbf{a}$ the occupied $(-1.1 \mathrm{~V})$ and $\mathbf{b}$ unoccupied $(1.4 \mathrm{~V})$ states and their corresponding $\mathrm{d} / / \mathrm{d} V$ maps (c) and (d), respectively. We observe two different types of defects located in the surface Te layer (type A) and in the subsurface Bi layer (Type B). The scale bar is $2 \mathrm{~nm}$. For the bias-dependent topographs and $d / / d V$ maps in the $[-1.5:+1.5 \mathrm{~V}]$ range see Supplementary Fig. 2.

observed on $\mathrm{d} / \mathrm{d} \mathrm{d} V$ maps taken at about $-0.08 \mathrm{~V}$ and attributed to $\mathrm{Bi}_{\mathrm{Mn}}{ }^{65}$. We do not observe such a feature in our $\mathrm{d} / / \mathrm{d} V$ measurements. Nevertheless, as shown in Supplementary Information, the presence of $\mathrm{Bi}_{\mathrm{Mn}}$ atoms is indeed confirmed by our structure refinement. The results of our structure characterizations are in agreement with the previous $X-$ ray $^{29}$ and neutron ${ }^{51,58}$ diffraction studies, as well as with the conclusions based on electron energy loss spectroscopy and transmission electron microscopy ${ }^{12,56}$. It is not surprising that the signatures of $\mathrm{Bi}_{\mathrm{Mn}}$ and $\mathrm{Mn}_{\mathrm{Bi}}$ lying in the fourth and sixth layers are not clearly seen on topographies as the tunneling probability depends drastically of the tip-sample distance. In addition, the corresponding features (whose extension should be about several lattice parameters as a minimum) may laterally overlap with each other, making them hardly distinguishable. Nevertheless, based on the agreement between the concentrations of the $\mathrm{Mn}_{\mathrm{Bi}}$ substitutions measured by STM and XRD one can conclude that these and $\mathrm{Bi}_{\mathrm{Mn}}$ appear already in bulk before the crystal cleavage.

\section{ARPES and STS measurements}

Let us now discuss the surface electronic structure of our $\mathrm{MnBi}_{2} \mathrm{Te}_{4}$ samples based on the results of the laser-ARPES and STS measurements performed in the AFM state (Fig. 4). In ARPES, the linearly dispersing topological surface state (TSS) is clearly visible in Fig. 4a. The energy distribution curve (EDC) at the $\bar{\Gamma}$ point (red dotted curve in Fig. 4b) presents a minimum at binding energies of about $0.27 \mathrm{eV}$, indicating the presence of a gap at the DP. According to the EDC fitting, the gap value is about $55 \mathrm{meV}$, similar to what has been reported previously ${ }^{7,41,48}$. The secondderivative representation (Fig. 4e) provides a clear illustration of the gapped Dirac cone. STS data recorded with high $k_{\|}$resolution (see Methods for more information) at $1.2 \mathrm{~K}$ (Fig. $4 \mathrm{f}$ ), i.e., well below the Néel temperature, show a $d / / d V$ spectrum featuring a local minimum near the expected DP position. This is compatible with the gap of the order of $50 \mathrm{meV}$ in agreement with the result of the EDC analysis shown in Fig. 4b. Even though the $\mathrm{d} / \mathrm{d} V \mathrm{~V}$ signal does not vanish at the (gapped) DP, the $\mathrm{d} / / \mathrm{d} V$ map at $-0.27 \mathrm{~V}$ displayed in Supplementary Fig. 4 is featureless and homogeneous, meaning that only a background signal is detected, i.e., no states are present. Similar featureless maps are observed in a range of $-0.27 \pm 0.02 \mathrm{~V}$, in line with the DP gap of about $50 \mathrm{meV}$ observed in ARPES (Fig. 4a, b, e). For another sample, however, the laser-ARPES reveals a TSS with a substantially reduced gap, of the order of $20 \mathrm{meV}$ (Fig. 4c, d, g). Indeed, the $\bar{\Gamma}$ point EDC (Fig. 4d) shows an apparent peak near the expected DP position. Such an EDC spectrum is a consequence of the overlap of the two (unresolved, but still present) edges of the gapped TSS, which, according to the EDC fitting, results in an asymmetric peak. The signatures of such behavior are also seen in the STS (note that the samples studied in ARPES and STM/S are different, although they are from the same batch), showing a peak at $-0.29 \mathrm{~V}$ (Fig. 4h), consistent with the presence of the unresolved spectral features revealed by the EDC fitting at $\bar{\Gamma}$. In this case, as can be seen in Supplementary Fig. 5, the $\mathrm{d} / \mathrm{d} V$ map at $-0.30 \mathrm{~V}$ exhibits a stronger signal, as a result of the contribution of the edges of the Dirac cone states observed also in the EDC (Fig. 4d). The modulation of the $d / / d V$ signal is more affected in this case by the presence of the deep defects (see Supplementary Fig. 5c). However, the overall shape of the spectra shown in Fig. $4 f, h$ is independent of point defects (see Supplementary Figs. 4, 5, respectively).

\section{DISCUSSION}

We now discuss the possible origin of the DP gap size variation from sample to sample and within one sample. Well-defined dispersion lines observed with laser-ARPES indicate reasonably good quality of the crystal surface, in agreement with our STM observations. Therefore, the surface SL crystal structure is largely similar to that of SLs in bulk and thus the near-surface magnetic structure should be the same as in bulk too, which has been recently confirmed using magnetic force microscopy ${ }^{66}$. Thus, the variation (or a complete closing ${ }^{42-47}$ ) of the DP gap does not seem to come from a radical change of the magnetism at the surface. Neither it comes from some severe surface crystal structure degradation, not observed for the $\mathrm{MnBi}_{2} \mathrm{Te}_{4}$ single crystals in ultrahigh vacuum. From the available STM and XRD evidence, apart from the unavoidable steps at the surface, the only significant structural imperfections of $\mathrm{MnBi}_{2} \mathrm{Te}_{4}$ are related to point defects, caused by cation ( $\mathrm{Mn}-\mathrm{Bi}$ ) intermixing.

The evidence presented here and in the literature indicates that these defects are formed in the bulk of the sample during its growth and then naturally find themselves near the surface because of the crystal cleavage before the ARPES or STM/S measurements. The role of these defects for magnetic properties of $\mathrm{MnBi}_{2} \mathrm{Te}_{4}$ and related compounds is being discussed currently ${ }^{52,53,67,68}$. Recent high-field magnetization measurements show that reaching the saturation magnetization of $\mathrm{MnBi}_{2} \mathrm{Te}_{4}$ (corresponding to about $4.6 \mu_{B}$ per $\mathrm{Mn}$ ) requires very large external magnetic fields of about $60 \mathrm{~T}^{53}$, while many previous studies revealed an incomplete saturation, 3-3.8 $\mu_{B}$ per Mn at about $6-7 T^{7,12,13,15,19,69,70}$. The reason for this has been found to be a "ferrimagnetic" structure of the septuple layer block, in which the local moments of the $\mathrm{Mn}_{\mathrm{Bi}}$ defects are coupled antiparallel to those of the central Mn layer ${ }^{51,71}$. This is completely analogous to what is observed in $\mathrm{MnSb}_{2} \mathrm{Te}_{4}{ }^{51,52}$, which is a related isostructural compound ${ }^{4,24,25}$. The essential difference between $\mathrm{MnBi}_{2} \mathrm{Te}_{4}$ and $\mathrm{MnSb}_{2} \mathrm{Te}_{4}$ is a more pronounced cation intermixing in the latter ${ }^{51,52}$, meaning a larger number of the $\mathrm{Mn}$ atoms at the $\mathrm{Sb}$ sites $\left(\mathrm{Mn}_{\mathrm{sb}}\right)$. Recent neutron diffraction measurements ${ }^{51,52}$ have shown the AFM coupling between the central Mn layer and the $\mathrm{Mn}_{\mathrm{Sb}}$ atoms. Due to a large amount of $\mathrm{Mn}_{\mathrm{Sb}}$ atoms in $\mathrm{MnSb}_{2} \mathrm{Te}_{4}$, the magnetic moment per $\mathrm{Mn}$ atom at $6-7 \mathrm{~T}$ is only about $2 \mu_{B}^{53}$. 
(a)

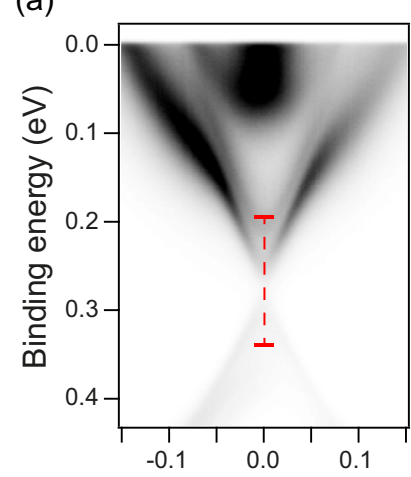

(e)

Wave vector $\left(\AA^{-1}\right)$

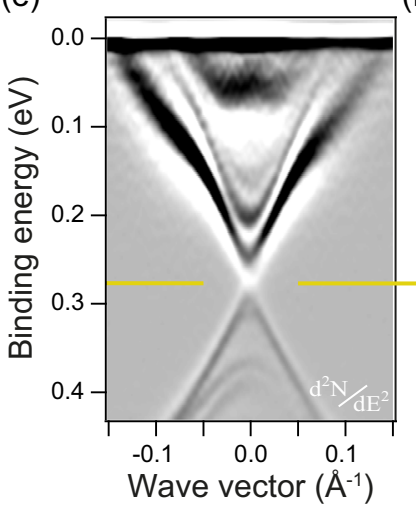

(b)

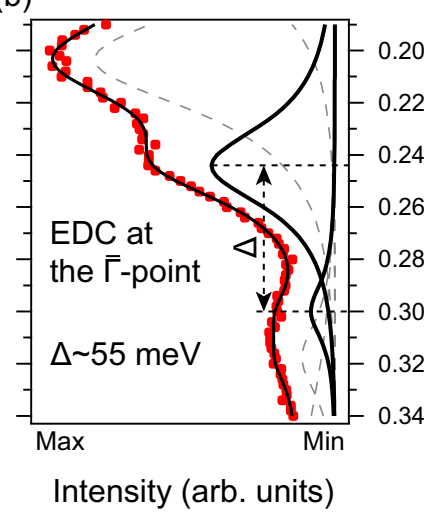

(f)

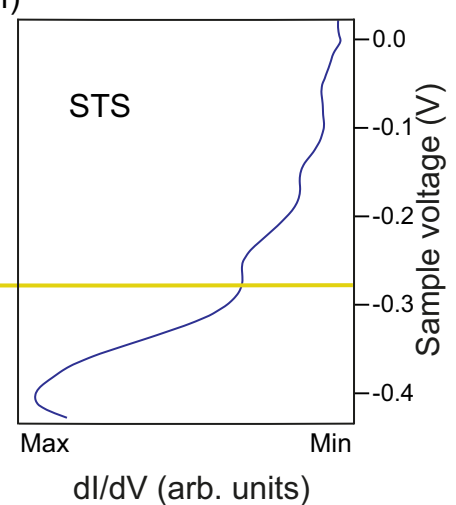

(c)

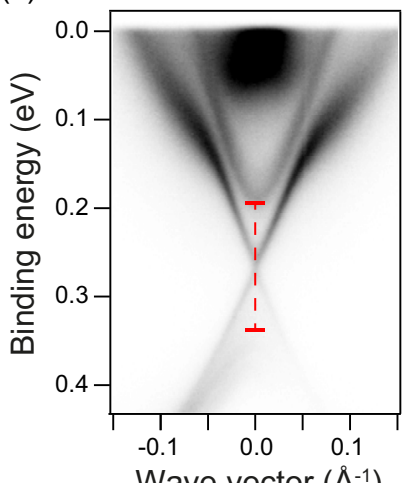

(d)

(g)

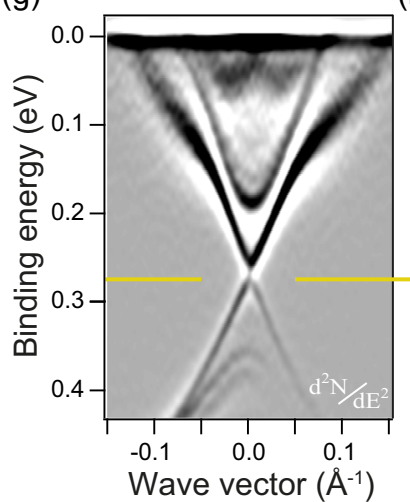

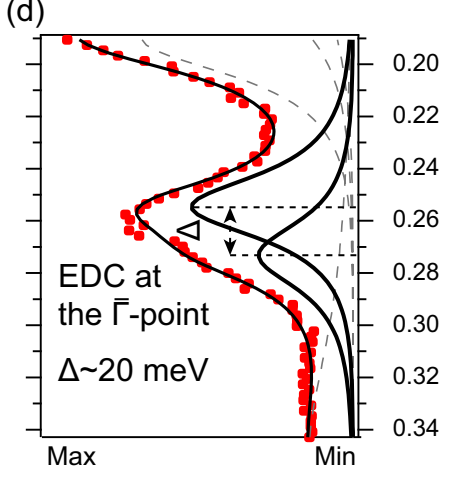

Intensity (arb. units)

(h)

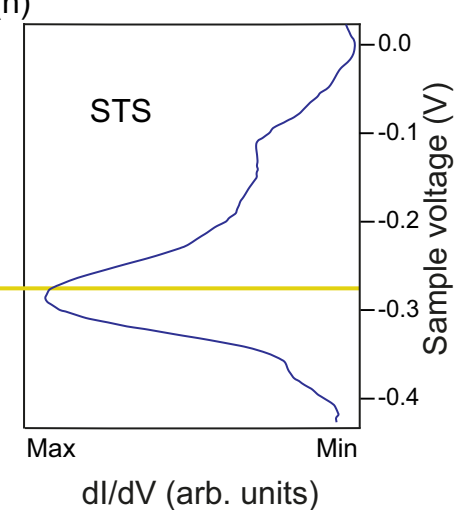

Fig. 4 Comparison of the ARPES and STS data for different Dirac point gaps. a, c Measured $\mathrm{MnBi}_{2} \mathrm{Te}_{4}(0001)$ ARPES dispersions corresponding to a larger (a) and smaller (c) DP gaps (measurements conditions: photon energy $h v=6.3 \mathrm{eV}$; temperature $T=10 \mathrm{~K}$ ). (b,d) Measured (red points) and fitted (solid black curves) $\bar{\Gamma}$-point EDCs acquired at binding energies close to the gapped DP with decomposition on the spectral components shown. The peaks corresponding to the upper and lower Dirac cone parts are shown by the bold solid black lines, while those of the bulk conduction band -- by the thin dashed gray lines. The binding energy intervals of the presented EDCs correspond to the intervals marked in (a, c) by vertical red lines. EDCs fitting yields the DP gap values of $\Delta \sim 55 \mathrm{meV}$ (b) and $\Delta \sim 20 \mathrm{meV}$ (d). e, $\mathbf{g}$ Secondderivative $\left(\mathrm{d}^{2} \mathrm{~N} / \mathrm{d} E^{2}\right)$ representation of the data shown in $(\mathbf{a}, \mathbf{c})$, respectively, providing better visualization of the larger $(\mathbf{e})$ and smaller $(\mathbf{g})$ DP gaps. (f, h) Spatially averaged tunneling conductance spectra showing a clear dip (f) and peak (h) at the expected energy position of the gapped DP. The spectra shown in (f, h) are compatible with larger $(\sim 50 \mathrm{meV})$ and smaller $(<20 \mathrm{meV}) \mathrm{DP}$ gaps, respectively. The horizontal yellow lines show the correspondence between the ARPES and STS spectra. The $d / / d V$ curves in (f, $\mathbf{h}$ ) are shown in a wider bias voltage range in Supplementary Figs. 4, 5, respectively. The ARPES data in (a) and (c) correspond to two different samples, while the STS data in (f) and (g) have been acquired from yet another sample, but after different cleavages.

Therefore, similarly to $\mathrm{MnBi}_{2} \mathrm{Te}_{4}$, very strong fields of up to $60-70 \mathrm{~T}$ are needed to overcome the intrablock AFM coupling and fully polarize the SLs. In the $\mathrm{Mn}\left(\mathrm{Bi}_{1-x} \mathrm{Sb}_{x}\right)_{2} \mathrm{Te}_{4}$ solid solutions, the $M(H)$ behavior, observed in $\mathrm{MnSb}_{2} \mathrm{Te}_{4}$, continuously evolves into that of $\mathrm{MnBi}_{2} \mathrm{Te}_{4}{ }^{15,72}$. The latter facts strongly point towards the ferrimagnetic structure of the $\mathrm{MnBi}_{2} \mathrm{Te}_{4}$ SLs as well.

The ferrimagnetic structure, along with the presence of $\mathrm{Bi}_{M n}$ in the Mn layer (as found by our structure refinement as well as in refs. ${ }^{29,51,58}$ ), is expected to significantly reduce the effective magnetization of each individual SL block of $\mathrm{MnBi}_{2} \mathrm{Te}_{4}$. Eventually, at the surface, this should cause a decrease in the DP gap size. However, an approximately $20 \%$ decrease of magnetization of each SL, which can be expected based on our XRD data, can hardly explain the DP gap size reduction by at least a factor of 2, as we observe in ARPES and STS. The reason why the cation intermixing should strongly affect the DP gap size becomes clear when the real space TSS distribution is analyzed. As it is shown in Fig. $5 \mathrm{a}$, the weight of the TSS in the Te-Bi-Te trilayers of the surface $\mathrm{SL}$ is much larger than in the Te-Mn-Te trilayer. Thus, due to the $\mathrm{Mn}-\mathrm{Bi}$ intermixing, the magnetization of $\mathrm{Mn}_{\mathrm{Bi}}$, counteracting the effect of the central layer Mn atoms, is introduced exactly in the regions of the TSS predominant localization. In turn, the central $\mathrm{Mn}$ layer, where the TSS weight is small, becomes slightly "magnetization depleted" due to the $\mathrm{Bi}$ atoms incorporation.
Depending on the intermixing levels the cooperation of these two factors may result in a significant reduction of the size of the DP gap or even in its almost complete shrinking, a phenomenon that has been observed ${ }^{42-47}$, but not satisfactorily explained up to now.

To confirm the above-suggested scenario of the DP gap reduction, we have performed fully-relativistic DFT surface electronic structure calculations of $\mathrm{MnBi}_{2} \mathrm{Te}_{4}(0001)$ (see Methods section for the calculation details). It can be seen in Fig. $5 \mathrm{~b}$ that the pristine $\mathrm{MnBi}_{2} \mathrm{Te}_{4}$ surface features the DP gap of $90 \mathrm{meV}$, in agreement with the previous calculations ${ }^{7,8}$. Then, when a pair of $\mathrm{Mn}$ and $\mathrm{Bi}$ atoms are exchanged so that the $\mathrm{Mn}_{\mathrm{Bi}}$ atom goes in the subsurface atomic layer, the DP gap appears to be reduced by about 2.5 times, i.e., to $37 \mathrm{meV}$ (Fig. 5c). This already shows that the antiparallel alignment of the magnetic moments of $\mathrm{Mn}_{\mathrm{Bi}}$ (with respect to the central $\mathrm{Mn}$ layer) has an important effect on the $\mathrm{MnBi}_{2} \mathrm{Te}_{4}$ DP gap size. However, while the topological surface state charge density shows a local maximum around the subsurface Bi layer, the second Bi layer (i.e., the sixth atomic layer counting from the surface) carries a much larger weight of the state (Fig. 5a). Remarkably, introducing $\mathrm{Mn}_{\mathrm{Bi}}$ defect in the 6 th layer leads to almost complete shrinking of the DP gap (Fig. 5d), whose calculated value amounts to $3.5 \mathrm{meV}$, i.e., by about 25 times smaller than in the defectless case. These results provide 

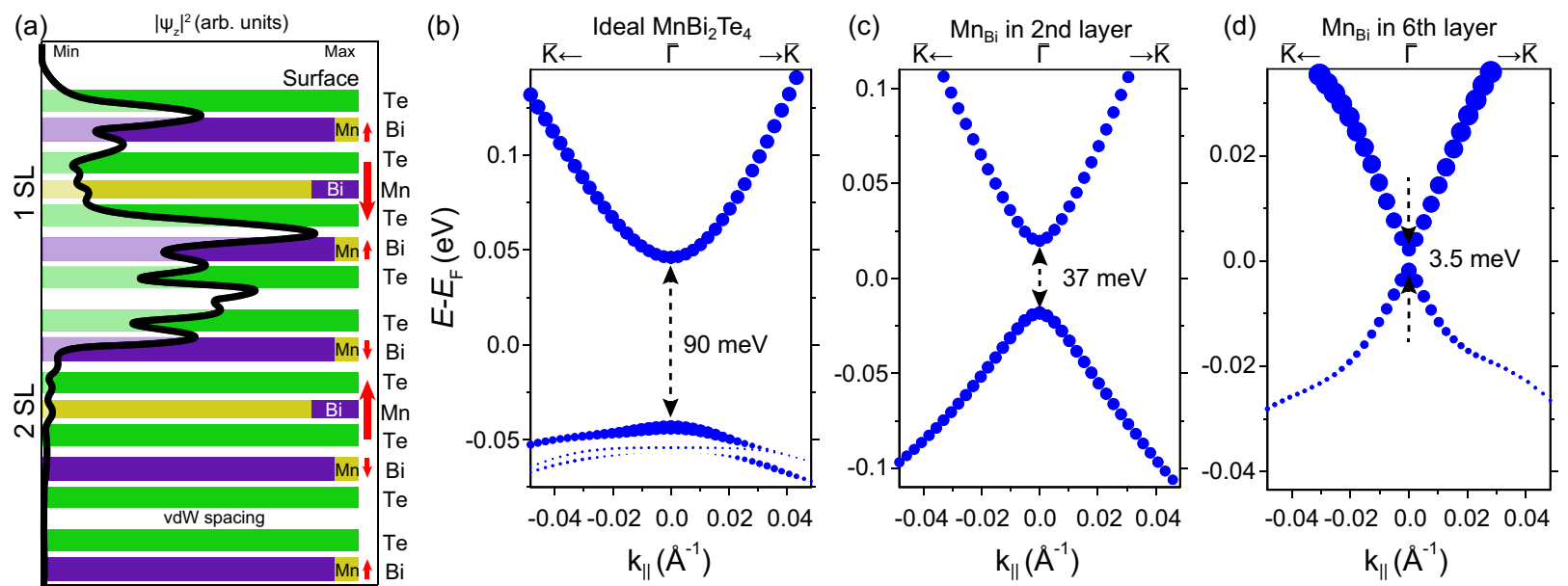

Fig. 5 DFT surface electronic structure calculations of ideal and defective $\mathbf{M n B i}_{\mathbf{2}} \mathbf{T e}_{\mathbf{4}}$. a Illustration of the TSS real space distribution at the $\mathrm{MnBi}_{2} \mathrm{Te}_{4}(0001)$ surface. The $\left|\Psi_{z}\right|^{2}$ profile corresponds to the band structure shown in panel $\mathbf{b}$. The color coding for the atoms sorts is the same as in Fig. 1a. Unlike in the case of the ideal structure (Fig. 1a), the $\mathrm{Mn}$-Bi intermixing leads to the appearance of the $\mathrm{Mn}_{\mathrm{Bi}}$ magnetic moments in the Bi layers that are coupled antiparallel to those in the central Mn layer of the same SL. The Mn $\mathrm{Bi}_{\mathrm{Bi}}$ magnetic moments thus turn out to be located in the regions with a high weight of the TSS, strongly counteracting the effect from the magnetization of the central Mn layer, where the TSS weight is low. This is expected to lead to a strong reduction of the DP gap. The latter is illustrated in panels $(\mathbf{b}-\mathbf{d})$, where the $\mathrm{MnBi}_{2} \mathrm{Te}_{4}$ surface electronic structure in the defectless case (b) is compared to those with $\mathrm{Mn}$-Bi intermixing when $\mathrm{Mn}_{\mathrm{Bi}}$ defect locates in the second (c) and sixth (d) atomic (Bi) layers counting from the surface (see a). Note that only the topological surface state is shown, while the bulk-like bands are omitted. The energy axes scales in $(\mathbf{b}, \mathbf{c})$ and $(\mathbf{d})$ are different.

theoretical proof that the $\mathrm{Mn}_{\mathrm{Bi}}$ defects can cause a strong reduction of the $\mathrm{MnBi}_{2} \mathrm{Te}_{4}$ DP gap due to the ferrimagnetic structure of the $\mathrm{SL}^{51-53}$ and predominant localization of the topological surface state in the Bi layers of the surface SL block.

A recent study reports ${ }^{52}$ that the degree of the cation intermixing in $\mathrm{MnSb}_{2} \mathrm{Te}_{4}$ may be varied by changing the growth temperature. In $\mathrm{MnBi}_{2} \mathrm{Te}_{4}$, the $\mathrm{Mn}-\mathrm{Bi}$ intermixing should be sensitive to the growth temperatures and starting compositions too and, therefore, one can expect that the degree of it may differ from sample to sample or may even experience certain variations in the same crystal. Indeed, the per layer concentrations of the $\mathrm{Mn}_{\mathrm{Bi}}$ atoms in the single crystal samples by different groups are reported to range from 2.5 to $5 \%(4.6-5.7 \%)$ as estimated based on the STM measurements ${ }^{13,62,63,65}$ (XRD measurements in this work and ref. ${ }^{29}$ ). Besides, our STM measurements indicate the fluctuation of the $\mathrm{Mn}_{\mathrm{Bi}}$ concentration within one sample (Supplementary Fig. 6). It seems like such variations of the defects concentrations may also affect the free carriers concentration and the value of the Néel temperature ${ }^{7,12,13,29}$, that slightly varies from sample to sample within the $24-25.4 \mathrm{~K}$ range (Supplementary Fig. 9 and the corresponding note). These arguments provide a plausible explanation of the reason for the observation of the different DP gap values in different samples.

Let us now discuss the here proposed DP gap reduction scenario in the context of the temperature-induced transition into the paramagnetic phase. When the Dirac cone is nearly gapless, then no strong changes are expected to be seen in ARPES upon heating above the Néel temperature. However, when the DP gap is sizable, the magnetic nature of the here proposed mechanism implies that it should close in the high-temperature magnetically disordered state. While the early synchrotron ARPES studies reported ${ }^{7,12,14}$ that the DP gap in $\mathrm{MnBi}_{2} \mathrm{Te}_{4}$ persists well above the Néel temperature, recent laser-ARPES data show about $40 \%$ reduction of the DP gap (from 65 to $40 \mathrm{meV}$ ) upon heating from below $T_{N}$ up to $35 \mathrm{~K}^{73}$. Incomplete closing of the gap seems to be consistent with strong short-range order effects that persist in $\mathrm{MnBi}_{2} \mathrm{Te}_{4}$ up to about $50-60 \mathrm{~K}$, as observed by electron spin resonance, ferromagnetic resonance, and antiferromagnetic resonance experiments $s^{7,74,75}$. The measured magnetization data ${ }^{53}$, revealing that $\mathrm{MnBi}_{2} \mathrm{Te}_{4}$ is not in the paramagnetic limit even at
$T \approx 50 \mathrm{~K}$, confirms this observation. Such a behavior is also consistent with the strong spin fluctuation-driven spin scattering above $T_{N}$ found in a previous magneto-transport study of $\mathrm{MnBi}_{2} \mathrm{Te}_{4}$ in ref. ${ }^{12}$. Beyond $50-60 \mathrm{~K}$, a large anisotropy of the $\mathrm{Mn}$ spin relaxation rate in the paramagnetic state of $\mathrm{MnBi}_{2} \mathrm{Te}_{4}{ }^{7,74}$ may give rise to an instantaneous (on the timescale of electron spin resonance) out-of-plane magnetic field at the surface, preventing the gap to close even at $T>T_{N}$ on the much faster timescale of the ARPES experiment.

The latter interpretation of the ARPES data implies that the DP gap closing should in principle be observable with other techniques. Recently, local measurements with point-contact tunneling spectroscopy have allowed detection of the magnetic gap at the DP of $\mathrm{MnBi}_{2} \mathrm{Te}_{4}$ at some surface locations ${ }^{76}$. Although in other surface areas there was no gap detected by the same technique, this does not contradict the here proposed scenario based on the crucial role of the $\mathrm{Mn}-\mathrm{Bi}$ intermixing, since the degree of the intermixing may vary across the surface. Indeed, as we have written above, our STM measurements show that the $\mathrm{Mn}_{\mathrm{Bi}}$ concentration fluctuates across the surface at the $100 \times$ $100 \mathrm{~nm}^{2}$ scale, at least judging by the $\mathrm{Mn}_{\mathrm{Bi}}$ concentration in the subsurface layers. We have also found that cleaving the sample exposes a different surface with the same property, i.e., a fluctuating concentration of $\mathrm{Mn}_{\mathrm{Bi}}$ across the surface. Thus, a larger (smaller) average concentration of $\mathrm{Mn}_{\mathrm{Bi}}$ in XRD or any other integral technique will not straightforwardly translate into a smaller (larger) DP gap in STS: the size of the latter will be a rather local property as compared to the scale of the sample size. Moreover, it might well be a local property even in $\mu$-laser-ARPES, although at a larger scale (the light spot is about $5 \mu \mathrm{m}$ ). Indeed, the ARPES mapping of the $\mathrm{MnBi}_{2} \mathrm{Te}_{4}$ surface shows that the electronic structure is inhomogeneous on the scale of 100-150 $\mu \mathrm{m}$ (see Supplementary Fig. 4 of ref. ${ }^{41}$ ). Ideally, an in situ study of the very same surface and its very same local area by low-temperature $\mu$-laser-ARPES and low-temperature STM/S in the same instrumental setup is required. However, given the highly different spatial scales of the ARPES and STM, this appears to be hardly feasible. Our results thus highlight a necessity to suppress the cation (Mn-Bi) intermixing in $\mathrm{MnBi}_{2} \mathrm{Te}_{4}$ thus minimizing the number of $\mathrm{Mn}_{\mathrm{Bi}}$, which is a crucial task for the nearest future 
studies. Improving the structural quality of $\mathrm{MnBi}_{2} \mathrm{Te}_{4}$ up to the level of the state-of-the-art samples of its parent compound $\mathrm{Bi}_{2} \mathrm{Te}_{3}{ }^{64,77}$ will hopefully allow getting rid of the DP gap issue in this AFM TI.

In conclusion, we have experimentally studied the crystalline and electronic structure of the (0001) surface of the AFM TI $\mathrm{MnBi}_{2} \mathrm{Te}_{4}$ using STM/S, micro $(\mu)$-laser ARPES, and first-principles calculations. On a large scale, the surface appears to be atomically flat, with several hundred nanometer wide terraces, separated by septuple layer high steps. On the atomic scale, a well-defined hexagonal lattice is detected with a $(1 \times 1)$ periodicity, i.e., no reconstruction or degradation occurs upon cleavage, as generally expected for a 2D van der Waals material. Further, we clearly observe two kinds of spectroscopic features on the topographic STM images. Namely, we distinguish (i) circular protrusions stemming from the $\mathrm{Bi}$ atoms at the surface $\mathrm{Te}$ sites and (ii) triangular depressions due to the $\mathrm{Mn}$ atoms at the subsurface $\mathrm{Bi}$ sites. The presence of the $\mathrm{Mn}$ atoms in the subsurface Bi layer indicates that they are located in the sixth $\mathrm{Bi}$ layer, too, while $\mathrm{Bi}$ atoms, in turn, occupy Mn positions in the fourth layer (cation intermixing), which is strongly supported by the results of our $\mathrm{X}$-ray diffraction data refinement.

Our low-temperature STS/ $\mu$-laser-ARPES experiments reveal that the size of the Dirac point gap in the topological surface state differs for different cleavages/samples. We attribute this behavior to the effect of the spatially inhomogeneous cation (Mn-Bi) intermixing, which affects the distribution of $\mathrm{Mn}$ atoms and, according to the recent high-field magnetization measurements ${ }^{53}$, leads to a deviation of the $\mathrm{MnBi}_{2} \mathrm{Te}_{4}$ septuple layer magnetic structure from the ideal ferromagnetic to a ferrimagnetic one. The latter structure along with the predominant real-space localization of the topological surface state around the Bi layers of the topmost septuple layer leads to a dramatic reduction of the Dirac point gap size, as revealed by our first-principles electronic structure calculations. A variation of the degree of defectness should lead to a different exchange splitting of the Dirac point for different samples or sample cleavages.

\section{METHODS}

\section{Crystal growth}

The bulk $\mathrm{MnBi}_{2} \mathrm{Te}_{4}$ single crystals were grown by the modified Bridgman method. To perform a careful refinement of the $\mathrm{MnBi}_{2} \mathrm{Te}_{4}$ crystal structure, we synthesized polycrystalline single-phase samples, that contained no $\mathrm{MnTe}$ or any other phases. The synthesis was carried out in sealed quartz ampoules by melting elements taken in stoichiometric ratios. Samples of the polycrystalline alloy were ground, pressed into pellets, and annealed at $575^{\circ} \mathrm{C}$. This process was repeated three times with a total annealing time of $750 \mathrm{~h}$. The diffraction pattern obtained and the corresponding structure refinement are presented in the Supplementary Information.

\section{STM/S measurements}

STM/S measurements were performed on a custom-designed ultra-high vacuum (UHV) system equipped with a low-temperature scanning tunneling microscope. The crystal was cleaved by Nitto tape in situ at room temperature and directly transferred to the STM. The base pressure during the experiments was $2 \times 10^{-10} \mathrm{mbar}$. STM images were recorded in constant current mode and the differential conductance $(\mathrm{d} / / \mathrm{d} V$ spectra were taken using a lock-in amplifier $(f=763.7 \mathrm{~Hz})$ at 4.7 and $1.2 \mathrm{~K}$. We use freshly $\mathrm{Ar}^{+}$sputtered polycrystalline $\mathrm{W}$ tips, which we carefully prepare on gold to have a blunt apex. As a result, $\mathrm{d} / / \mathrm{d} V$ spectra reflect the k-integrated LDOS in a small $\Delta k_{\|}$region comparable with the EDC measured with ARPES at the $\Gamma$ point. The images were processed using the WSxM software ${ }^{78}$.

\section{ARPES measurements}

The ARPES measurements were carried using $\mu$-laser with improved angle and energy resolution and a laser beam with a spot diameter around $5 \mu \mathrm{m}$, using a Scienta R4000 electron energy analyzer with an incidence angle of $50^{\circ}$ relatives to the surface normal. The measurements were performed using $p$-polarized laser radiation with photon energy $h v=6.3 \mathrm{eV}$ at a temperature of $10 \mathrm{~K}$.

\section{Resistivity measurements}

Resistivity measurements were done with a standard four-probe ac technique using a low-frequency $(f \approx 20 \mathrm{~Hz})$ lock-in amplifier. Contacts were attached with conducting graphite paste.

\section{DFT calculations}

Electronic structure calculations were carried out within the density functional theory using the projector augmented-wave (PAW) method ${ }^{79}$ as implemented in the VASP code ${ }^{80,81}$. The exchange-correlation energy was treated using the generalized gradient approximation ${ }^{82}$. The energy cutoff for the plane-wave expansion was set to $270 \mathrm{eV}$. The $\mathrm{Mn} 3 d$-states were treated employing the GGA $+U$ approach ${ }^{83}$ within the Dudarev scheme ${ }^{84}$. The $U_{\text {eff }}=U-J$ value for the $M n 3 d$-states was chosen to be equal to $5.34 \mathrm{eV}$, as in previous works $\mathrm{s}^{2-5,7,8,24,85-87}$.

STM/S simulations were performed using the Tersoff-Hamann approximation. We have chosen a $(5 \times 3 \sqrt{3})$ rectangular cell $(21.68 \AA \times 22.53 \AA$; about 420 atoms), containing two $\mathrm{MnBi}_{2} \mathrm{Te}_{4}$ septuple layers and a vacuum layer with a thickness of no less than $10 \AA$. Structural optimizations were performed using a conjugate-gradient algorithm and a force tolerance criterion for convergence of $0.01 \mathrm{eV} / \AA$. The $\bar{\Gamma}$-centered $k$-point meshes of $2 \times 2 \times 1$ and $3 \times 3 \times 1$ were used to sample the $2 \mathrm{D}$ Brillouin zone for the relaxations and static calculations, respectively. In order to describe the van der Waals interactions, we made use of the DFT-D3 ${ }^{88,89}$ approach. Spin-orbit coupling was neglected.

In the surface electronic structure calculations, the Hamiltonian contained scalar relativistic corrections, and the spin-orbit coupling was taken into account by the second variation method ${ }^{90}$. The 6-SL-thick slab and the $(3 \times 3)$ in-plane supercell have been chosen (378 atoms). The $\bar{\Gamma}$-centered $k$-point mesh of $3 \times 3 \times 1$ has been used. We have compared three following cases: (i) ideal $\mathrm{MnBi}_{2} \mathrm{Te}_{4}$, and $\mathrm{MnBi}_{2} \mathrm{Te}_{4}$ with one $\mathrm{Mn}_{\mathrm{Bi}}$ substitution in the (ii) second and (iii) sixth atomic layers ( $11 \%$ per layer). In the latter two cases, the substituted $\mathrm{Bi}$ atom has been placed in the central $(\mathrm{Mn})$ layer of the surface SL. The defects have only been introduced in the surface SL because the topological surface state charge density is largest there (see Fig. 5a). Structural relaxations due to the introduction of the $\mathrm{Mn}-\mathrm{Bi}$ intermixing were neglected. The ferrimagnetic structure of the surface SL has been assumed in which the local moment of the $M n_{B i}$ defect is coupled antiparallel to those of the central Mn layer ${ }^{51-53}$.

\section{DATA AVAILABILITY}

The authors declare that the data supporting the findings of this study are available within the paper and its supplementary information files.

\section{CODE AVAILABILITY}

The codes used to perform data analysis are available on request from the authors.

Received: 1 June 2021; Accepted: 10 December 2021; Published online: 14 January 2022

\section{REFERENCES}

1. Tokura, Y., Yasuda, K. \& Tsukazaki, A. Magnetic topological insulators. Nat. Rev. Phys. 1, 126-143 (2019).

2. Otrokov, M. M. et al. Highly-ordered wide bandgap materials for quantized anomalous Hall and magnetoelectric effects. 2D Mater. 4, 025082 (2017).

3. Otrokov, M. M. et al. Magnetic extension as an efficient method for realizing the quantum anomalous Hall state in topological insulators. JETP Lett. 105, 297-302 (2017).

4. Eremeev, S. V., Otrokov, M. M. \& Chulkov, E. V. Competing rhombohedral and monoclinic crystal structures in $\mathrm{MnPn}_{2} \mathrm{Ch}_{4}$ compounds: an ab-initio study. $J$. Alloys Compd. 709, 172-178 (2017).

5. Hirahara, T. et al. Large-gap magnetic topological heterostructure formed by subsurface incorporation of a ferromagnetic layer. Nano Lett. 17, 3493-3500 (2017).

6. Hagmann, J. A. et al. Molecular beam epitaxy growth and structure of selfassembled $\mathrm{Bi}_{2} \mathrm{Se}_{3} / \mathrm{Bi}_{2} \mathrm{MnSe}_{4}$ multilayer heterostructures. N. J. Phys. 19, 085002 (2017). 
7. Otrokov, M. M. et al. Prediction and observation of an antiferromagnetic topological insulator. Nature 576, 416-422 (2019).

8. Otrokov, M. M. et al. Unique thickness-dependent properties of the van der Waals interlayer antiferromagnet $\mathrm{MnBi}_{2} \mathrm{Te}_{4}$ films. Phys. Rev. Lett. 122, 107202 (2019).

9. $\mathrm{Li}$, J. et al. Intrinsic magnetic topological insulators in van der Waals layered $\mathrm{MnBi}_{2} \mathrm{Te}_{4}$-family materials. Sci. Adv. 5, eaaw5685 (2019).

10. Zhang, D. et al. Topological axion states in the magnetic insulator $\mathrm{MnBi}_{2} \mathrm{Te}_{4}$ with the quantized magnetoelectric effect. Phys. Rev. Lett. 122, 206401 (2019).

11. Gong, Y. et al. Experimental realization of an intrinsic magnetic topological insulator. Chin. Phys. Lett. 36, 076801 (2019).

12. Lee, S. H. et al. Spin scattering and noncollinear spin structure-induced intrinsic anomalous Hall effect in antiferromagnetic topological insulator $\mathrm{MnBi}_{2} \mathrm{Te}_{4}$. Phys. Rev. Res. 1, 012011 (2019).

13. Yan, J.-Q. et al. Crystal growth and magnetic structure of $\mathrm{MnBi}_{2} \mathrm{Te}_{4}$. Phys. Rev. Mater. 3, 064202 (2019).

14. Vidal, R. C. et al. Surface states and Rashba-type spin polarization in antiferromagnetic $\mathrm{MnBi}_{2} \mathrm{Te}_{4}(0001)$. Phys. Rev. B 100, 121104 (2019).

15. Yan, J.-Q. et al. Evolution of structural, magnetic, and transport properties in $\mathrm{MnBi}_{2-x} \mathrm{Sb}_{x} \mathrm{Te}_{4}$. Phys. Rev. B 100, 104409 (2019).

16. Rienks, E. et al. Large magnetic gap at the Dirac point in $\mathrm{Bi}_{2} \mathrm{Te}_{3} / \mathrm{MnBi}_{2} \mathrm{Te}_{4}$ heterostructures. Nature 576, 423-428 (2019).

17. Chen, B. et al. Intrinsic magnetic topological insulator phases in the Sb doped $\mathrm{MnBi}_{2} \mathrm{Te}_{4}$ bulks and thin flakes. Nat. Commun. 10, 4469 (2019).

18. $\mathrm{Wu}$, J. et al. Natural van der Waals heterostructural single crystals with both magnetic and topological properties. Sci. Adv. 5, eaax9989 (2019).

19. Li, B. et al. Competing magnetic interactions in the antiferromagnetic topological insulator $\mathrm{MnBi}_{2} \mathrm{Te}_{4}$. Phys. Rev. Lett. 124, 167204 (2020).

20. $\mathrm{Hu}, \mathrm{C}$. et al. A van der Waals antiferromagnetic topological insulator with weak interlayer magnetic coupling. Nat. Commun. 11, 97 (2020).

21. Klimovskikh, I. I. et al. Tunable $3 \mathrm{D} / 2 \mathrm{D}$ magnetism in the $\left(\mathrm{MnBi}_{2} \mathrm{Te}_{4}\right)\left(\mathrm{Bi}_{2} \mathrm{~T}_{3}\right)_{m}$ topological insulators family. npj Quantum Mater. 5, 54 (2020).

22. $\mathrm{He}, \mathrm{K} . \mathrm{MnBi}_{2} \mathrm{Te}_{4}$-family intrinsic magnetic topological materials. npj Quantum Mater. 5, 90 (2020).

23. Hirahara, T. et al. Fabrication of a novel magnetic topological heterostructure and temperature evolution of its massive Dirac cone. Nat. Commun. 11, 4821 (2020).

24. Wimmer, S. et al. Mn-rich $\mathrm{MnSb}_{2} \mathrm{Te}_{4}$ : a topological insulator with magnetic gap closing at high Curie temperatures of 45-50 K. Adv. Mater. 33, 2102935 (2021).

25. Eremeev, S. et al. Topological magnetic materials of the $\left(\mathrm{MnSb}_{2} \mathrm{Te}_{4}\right) \cdot\left(\mathrm{Sb}_{2} \mathrm{Te}_{3}\right)_{n}$ van der Waals compounds family. J. Phys. Chem. Lett. 12, 4268 (2021).

26. Gao, A. et al. Layer hall effect in a $2 \mathrm{D}$ topological axion antiferromagnet. Nature 595, 521-525 (2021).

27. Lee, D. S. et al. Crystal structure, properties and nanostructuring of a new layered chalcogenide semiconductor, $\mathrm{Bi}_{2} \mathrm{MnTe}_{4}$. Cryst. Eng. Comm. 15, 5532-5538 (2013).

28. Aliev, Z. S. et al. Novel ternary layered manganese bismuth tellurides of the

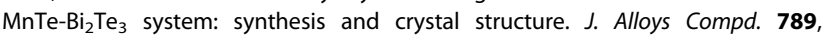
443-450 (2019).

29. Zeugner, A. et al. Chemical aspects of the candidate antiferromagnetic topological insulator $\mathrm{MnBi}_{2} \mathrm{Te}_{4}$. Chem. Mater. 31, 2795-2806 (2019).

30. $\mathrm{Li}$, B. et al. Quasi-two-dimensional ferromagnetism and anisotropic interlayer couplings in the magnetic topological insulator $\mathrm{MnBi}_{2} \mathrm{Te}_{4}$. Phys. Rev. B 104, L220402 (2021).

31. Mong, R. S. K., Essin, A. M. \& Moore, J. E. Antiferromagnetic topological insulators. Phys. Rev. B 81, 245209 (2010).

32. Fang, C., Gilbert, M. J. \& Bernevig, B. A. Topological insulators with commensurate antiferromagnetism. Phys. Rev. B 88, 085406 (2013).

33. Deng, Y. et al. Quantum anomalous Hall effect in intrinsic magnetic topological insulator $\mathrm{MnBi}_{2} \mathrm{Te}_{4}$. Science 367, 895-900 (2020).

34. Ge, J. et al. High-Chern-number and high-temperature quantum Hall effect without Landau levels. Natl Sci. Rev. 7, 1280-1287 (2020).

35. Liu, C. et al. Magnetic-field-induced robust zero Hall plateau state in $\mathrm{MnBi}_{2} \mathrm{Te}_{4}$ chern insulator. Nat. Commun. 12, 4647 (2021).

36. Deng, H. et al. High-temperature quantum anomalous Hall regime in a $\mathrm{MnBi}_{2} \mathrm{Te}_{4} /$ $\mathrm{Bi}_{2} \mathrm{Te}_{3}$ superlattice. Nat. Phys. 17, 36-42 (2020).

37. Liu, C. et al. Robust axion insulator and Chern insulator phases in a twodimensional antiferromagnetic topological insulator. Nat. Mater. 19, 522-527 (2020).

38. $\mathrm{Hu}, \mathrm{C}$. et al. Realization of an intrinsic ferromagnetic topological state in $\mathrm{MnBi}_{8} \mathrm{Te}_{13}$. Sci. Adv. 6, eaba4275 (2020).

39. Peng, Y. \& Xu, Y. Proximity-induced Majorana hinge modes in antiferromagnetic topological insulators. Phys. Rev. B 99, 195431 (2019).

40. Perez-Piskunow, P. M. \& Roche, S. Hinge spin polarization in magnetic topological insulators revealed by resistance switch. Phys. Rev. Lett. 126, 167701 (2021).

41. Estyunin, D. et al. Signatures of temperature driven antiferromagnetic transition in the electronic structure of topological insulator $\mathrm{MnBi}_{2} \mathrm{Te}_{4}$. APL Mater. 8, 021105 (2020).
42. Hao, Y.-J. et al. Gapless surface Dirac cone in antiferromagnetic topological insulator $\mathrm{MnBi}_{2} \mathrm{Te}_{4}$. Phys. Rev. X 9, 041038 (2019).

43. $\mathrm{Li}, \mathrm{H}$. et al. Dirac surface states in intrinsic magnetic topological insulators EuSn $\mathrm{As}_{2}$ and $\mathrm{MnBi}_{2} \mathrm{nTe}_{3 n+1}$. Phys. Rev. X 9, 041039 (2019).

44. Chen, Y. J. et al. Topological electronic structure and its temperature evolution in antiferromagnetic topological insulator $\mathrm{MnBi}_{2} \mathrm{Te}_{4}$. Phys. Rev. X 9, 041040 (2019).

45. Swatek, P. et al. Gapless Dirac surface states in the antiferromagnetic topological insulator $\mathrm{MnBi}_{2} \mathrm{Te}_{4}$. Phys. Rev. B 101, 161109 (2020).

46. Nevola, D. et al. Coexistence of surface ferromagnetism and a gapless topological state in $\mathrm{MnBi}_{2} \mathrm{Te}_{4}$. Phys. Rev. Lett. 125, 117205 (2020).

47. Yan, C. et al. Origins of electronic bands in the antiferromagnetic topological insulator $\mathrm{MnBi}_{2} \mathrm{Te}_{4}$. Phys. Rev. B 104, $\mathrm{L} 041102$ (2021).

48. Shikin, A. et al. Nature of the Dirac gap modulation and surface magnetic interaction in axion antiferromagnetic topological insulator $\mathrm{MnBi}_{2} \mathrm{Te}_{4}$. Sci. Rep. 10, 13226 (2020).

49. Zhang, X. \& Zhang, S.-C. Chiral interconnects based on topological insulators. In Proc. Micro-and Nanotechnology Sensors, Systems, and Applications IV 837309 (International Society for Optics and Photonics, 2012).

50. An, Y. et al. Nanodevices engineering and spin transport properties of $\mathrm{MnBi}_{2} \mathrm{Te}_{4}$ monolayer. npj Comput. Mater. 7, 45 (2021).

51. Riberolles, S. X. M. et al. Evolution of magnetic interactions in Sb-substituted $\mathrm{MnBi}_{2} \mathrm{Te}_{4}$. Phys. Rev. B 104, 064401 (2021).

52. Liu, Y. et al. Site mixing for engineering magnetic topological insulators. Phys. Rev. $X$ 11, 021033 (2021)

53. Lai, Y., Ke, L., Yan, J., McDonald, R. D. \& McQueeney, R. J. Defect-driven ferrimagnetism and hidden magnetization in $\mathrm{MnBi}_{2} \mathrm{Te}_{4}$. Phys. Rev. B 103, 184429 (2021).

54. Zhussupbekov, K. et al. Imaging and identification of point defects in $\mathrm{PtTe}_{2} . n p j 2 D$ Mater. Appl. 5, 14 (2021).

55. In a topographic image ( $\mathrm{d} / \mathrm{d} V$ map), the STM contrast is defined by occupied or unoccupied states in the energy interval $E_{F}-\mathrm{eV}$ or $E_{F}+\mathrm{eV}$ (at the applied bias voltage), respectively. Bright/dark contrast corresponds then high/low values of the local density of states.

56. Hou, F. et al. Te-vacancy-induced surface collapse and reconstruction in antiferromagnetic topological insulator $\mathrm{MnBi}_{2} \mathrm{Te}_{4}$. ACS Nano 14, 11262-11272 (2020).

57. Du, M.-H., Yan, J., Cooper, V. R. \& Eisenbach, M. Tuning fermi levels in intrinsic antiferromagnetic topological insulators $\mathrm{MnBi}_{2} \mathrm{Te}_{4}$ and $\mathrm{MnBi}_{4} \mathrm{Te}_{7}$ by defect engineering and chemical doping. Adv. Funct. Mater. 31, 2006516 (2021).

58. Ding, L. et al. Crystal and magnetic structures of magnetic topological insulators $\mathrm{MnBi}_{2} \mathrm{Te}_{4}$ and $\mathrm{MnBi}_{4} \mathrm{Te}_{7}$. Phys. Rev. B 101, 020412 (2020).

59. Hor, Y. S. et al. Development of ferromagnetism in the doped topological insulator $\mathrm{Bi}_{2-x} \mathrm{Mn}_{x} \mathrm{Te}_{3}$. Phys. Rev. B 81, 195203 (2010).

60. Lee, I. et al. Imaging dirac-mass disorder from magnetic dopant atoms in the ferromagnetic topological insulator $\mathrm{Cr}_{x}\left(\mathrm{Bi}_{0.1} \mathrm{Sb}_{0.9}\right)_{2-x} \mathrm{Te}_{3}$. Proc. Natl Acad. Sci. USA 112, 1316-1321 (2015).

61. Zhang, L. et al. Ferromagnetism in vanadium-doped $\mathrm{Bi}_{2} \mathrm{Se}_{3}$ topological insulator films. APL Mater. 5, 076106 (2017).

62. Yuan, Y. et al. Electronic states and magnetic response of $\mathrm{MnBi}_{2} \mathrm{Te}$ by scanning tunneling microscopy and spectroscopy. Nano Lett. 20, 3271 (2020).

63. Liang, Z. et al. Mapping Dirac fermions in the intrinsic antiferromagnetic topological insulators $\left(\mathrm{MnBi}_{2} \mathrm{Te}_{4}\right)\left(\mathrm{Bi}_{2} \mathrm{Te}_{3}\right)_{n}(n=0,1)$. Phys. Rev. B 102, 161115 (2020).

64. Netsou, A.-M. et al. Identifying native point defects in the topological insulator $\mathrm{Bi}_{2} \mathrm{Te}_{3}$. ACS Nano 14, 13172-13179 (2020).

65. Huang, Z., Du, M.-H., Yan, J. \& Wu, W. Native defects in antiferromagnetic topological insulator $\mathrm{MnBi}_{2} \mathrm{Te}_{4}$. Phys. Rev. Mater. 4, 121202 (2020).

66. Sass, P. M., Kim, J., Vanderbilt, D., Yan, J. \& Wu, W. Robust A-type order and spinflop transition on the surface of the antiferromagnetic topological insulator $\mathrm{MnBi}_{2} \mathrm{Te}_{4}$. Phys. Rev. Lett. 125, 037201 (2020).

67. Murakami, T. et al. Realization of interlayer ferromagnetic interaction in $\mathrm{MnSb}_{2} \mathrm{Te}_{4}$ toward the magnetic Weyl semimetal state. Phys. Rev. B 100, 195103 (2019).

68. Chen, W., Zhao Y., Yao, Q., Zhang, J. \& Liu, Q. Koopmans' theorem as the mechanism of nearly gapless surface states in self-doped magnetic topological insulators. Phys. Rev. B 103, L201102 (2021)..

69. $\mathrm{Li}, \mathrm{H}$. et al. Antiferromagnetic topological insulator $\mathrm{MnBi}_{2} \mathrm{Te}_{4}$ : synthesis and magnetic properties. Phys. Chem. Chem. Phys. 22, 556-563 (2020).

70. Jiao, F. et al. The layer-inserting growth of antiferromagnetic topological insulator $\mathrm{MnBi}_{2} \mathrm{Te}_{4}$ based on symmetry and its x-ray photoelectron spectroscopy. J. Supercond. Nov. Magn. 34, 1-9 (2021).

71. Liu, Y. \& Yan, J. "Mn/Bi site mixing and complex magnetic structure in $\mathrm{MnBi}_{2} \mathrm{Te}_{4}$." APS March Meeting 2021, https://meetings.aps.org/Meeting/MAR21/Session/ L45.10.

72. Lee, S. H. et al. Evidence for a magnetic-field-induced ideal type-ii Weyl state in antiferromagnetic topological insulator $\mathrm{Mn}\left(\mathrm{Bi}_{1-x} \mathrm{Sb}_{x}\right)_{2} \mathrm{Te}_{4}$. Phys. Rev. X 11, 031032 (2021) 
73. Shikin, A. M. et al. Sample-dependent Dirac-point gap in $\mathrm{MnBi}_{2} \mathrm{Te}_{4}$ and its response to applied surface charge: a combined photoemission and ab initio study. Phys. Rev. B 104, 115168 (2021).

74. Alfonsov, A. et al. Strongly anisotropic spin dynamics in magnetic topological insulators. Phys. Rev. B 103, L180403 (2021).

75. Alfonsov, A. et al. Magnetic field tuning of the spin dynamics in the magnetic topological insulators $\left(\mathrm{MnBi}_{2} \mathrm{Te}_{4}\right)\left(\mathrm{Bi}_{2} \mathrm{Te}_{3}\right)_{n}$. Phys. Rev. B 104, 195139 (2021).

76. Ji, H.-R. et al. Detection of magnetic gap in topological surface states of $\mathrm{MnBi}_{2} \mathrm{Te}_{4}$. Chin. Phys. Lett. 38, 107404 (2021).

77. Sessi, $P$. et al. Visualizing spin-dependent bulk scattering and breakdown of the linear dispersion relation in $\mathrm{Bi}_{2} \mathrm{Te}_{3}$. Phys. Rev. B 88, 161407 (2013).

78. Horcas, l. et al. WSXM: a software for scanning probe microscopy and a tool for nanotechnology. Rev. Sci. Instrum 78, 013705 (2007).

79. Blöchl, P. E. Projector augmented-wave method. Phys. Rev. B 50, 17953-17979 (1994).

80. Kresse, G. \& Furthmüller, J. Efficient iterative schemes for ab initio total-energy calculations using a plane-wave basis set. Phys. Rev. B 54, 11169-11186 (1996).

81. Kresse, G. \& Joubert, D. From ultrasoft pseudopotentials to the projector augmented-wave method. Phys. Rev. B 59, 1758-1775 (1999).

82. Perdew, J. P., Burke, K. \& Ernzerhof, M. Generalized gradient approximation made simple. Phys. Rev. Lett. 77, 3865-3868 (1996).

83. Anisimov, V. I., Zaanen, J. \& Andersen, O. K. Band theory and Mott insulators: Hubbard U instead of Stoner I. Phys. Rev. B 44, 943-954 (1991).

84. Dudarev, S. L., Botton, G. A., Savrasov, S. Y., Humphreys, C. J. \& Sutton, A. P. Electron-energy-loss spectra and the structural stability of nickel oxide: An LSDA +U study. Phys. Rev. B 57, 1505-1509 (1998).

85. Eremeev, S. V., Otrokov, M. M. \& Chulkov, E. V. New universal type of interface in the magnetic insulator/topological insulator heterostructures. Nano Lett. 18, 6521-6529 (2018).

86. Jahangirli, Z. A. et al. Electronic structure and dielectric function of $\mathrm{Mn}-\mathrm{Bi}-\mathrm{Te}$ layered compounds. J. Vac. Sci. B 37, 062910 (2019).

87. Petrov, E. K. et al. Domain wall induced spin-polarized flat bands in antiferromagnetic topological insulators. Phys. Rev. B 103, 235142 (2021)

88. Grimme, S., Antony, J., Ehrlich, S. \& Krieg, H. A consistent and accurate ab initio parametrization of density functional dispersion correction (DFT-D) for the 94 elements H-Pu. J. Chem. Phys. 132, 154104 (2010).

89. Grimme, S., Ehrlich, S. \& Goerigk, L. Effect of the damping function in dispersion corrected density functional theory. J. Comput. Chem. 32, 1456-1465 (2011).

90. Koelling, D. D. \& Harmon, B. N. A technique for relativistic spin-polarised calculations. J. Phys. C Sol. State Phys. 10, 3107 (1977).

\section{ACKNOWLEDGEMENTS}

The authors thank M. Ilyn, M.A. Valbuena, and S.V. Eremeev for stimulating discussions We acknowledge support by the Spanish Ministerio de Ciencia e Innovacion (Grant no. PID2019-103910GB-I00, PGC2018-093291-B-I00, PGC2018-097028-A-100, and PGC2018-098613-B-C21) and Saint Petersburg State University (project ID No. 73028629). IMDEA Nanociencia acknowledges support from the "Severo Ochoa" Program for Centres of Excellence in R\&D (MINECO, Grant SEV-2016-0686). M.G. has received financial support through the Postdoctoral Junior Leader Fellowship Program from "la Caixa" Banking Foundation. Z.S.A. and N.T.M. acknowledge the support of the Science Development Foundation under the President of the Republic of Azerbaijan
(Grant No. EIF-BGM-4-RFTF-1/2017-21/04/1-M-02). I.I.K., D.E., and A.M.S. acknowledge the support from the Russian Science Foundation (Grant No. 18-12-00062) and the Russian Foundation of Basic Researches (Grant No. 20-32-70179).

\section{AUTHOR CONTRIBUTIONS}

M.G. and M.M.O. contributed equally to this work. E.V.C., M.M.O., M.G., and R.M. conceived and designed the experiments. M.G. and P.C.A. performed the STM/S experiments and conducted the analysis in collaboration with A.L.V.d.P. and R.M ARPES measurements were done by I.I.K., D.E., and A.M.S. Z.S.A. and M.B.B. grew the crystal and powder samples. X-ray diffraction measurements and structure determination were carried out by I.R.A. Resistivity measurements were performed by N.T.M., N.A.A., and V.N.Z. The DFT calculations were performed by M.M.O. and A.A. and their analysis was made together with E.V.C. The paper was written by M.M.O. with contributions from M.G., A.A., and R.M. All the authors discussed the results and commented on the manuscript.

\section{COMPETING INTERESTS}

The authors declare no competing interests.

\section{ADDITIONAL INFORMATION}

Supplementary information The online version contains supplementary material available at https://doi.org/10.1038/s41535-021-00414-6.

Correspondence and requests for materials should be addressed to M. Garnica, M. M. Otrokov, E. V. Chulkov or R. Miranda.

Reprints and permission information is available at http://www.nature.com/ reprints

Publisher's note Springer Nature remains neutral with regard to jurisdictional claims in published maps and institutional affiliations.

Open Access This article is licensed under a Creative Commons Attribution 4.0 International License, which permits use, sharing, adaptation, distribution and reproduction in any medium or format, as long as you give appropriate credit to the original author(s) and the source, provide a link to the Creative Commons license, and indicate if changes were made. The images or other third party material in this article are included in the article's Creative Commons license, unless indicated otherwise in a credit line to the material. If material is not included in the article's Creative Commons license and your intended use is not permitted by statutory regulation or exceeds the permitted use, you will need to obtain permission directly from the copyright holder. To view a copy of this license, visit http://creativecommons. org/licenses/by/4.0/.

C The Author(s) 2022 